(REVIEW ARTICLE)

\title{
Use of pramipexole in neuropsychiatry
}

\author{
Anatolii Tsarkov* and Petro Petlovanyi \\ Department of Psychiatry, School of Medicine, University of Zambia (UNZA).
}

Publication history: Received on 28 July 2020; revised on 05 August 2020; accepted on 09 August 2020

Article DOI: https://doi.org/10.30574/wjarr.2020.7.2.0283

\begin{abstract}
Parkinson's disease (PD) is a progressive neurological disorder characterized by a large number of motor and nonmotor features that can impact on function to a variable degree. The syndrome of parkinsonism is recognized, which can become apparent with akinetic rigid disorders in other brain injuries and medication-induced parkinsonism. Genetic mutations or variants, neuroimaging abnormalities and other tests are potential biomarkers that may improve diagnosis and allow the identification of persons at risk. This literature review describes pathogenesis and clinical characteristics of PD with emphasis on modern treatment and possibilities of dopamine agonists, especially pramipexole. During pramipexole treatment of the patients with the early and late stages of PD, we can observe the reduction of expression of cognitive and affective (mood) disorders, the improvement of the quality of life and social functioning.
\end{abstract}

Keywords: Parkinson's disease; Neurodegenerative disease; Neuropsychiatry; Psychopharmacology; Pramipexole; Depression

\section{Introduction}

One of the most prevailing and progressive neurodegeneration among the patients of elderly age (1-2\% in population older than 65 years) is Parkinson's disease (PD), which represents special interest to modern pharmacotherapy, which is aimed at the uncovering of key pathobiochemical mechanisms of brain's lesion [1], [2]. The prevalence of PD in Zambia is unknown, but it is the third of the most often disease after epilepsy and stroke [3]. Except PD, which is connected mainly with neurodegeneration, the syndrome of parkinsonism is highlighted, which can become apparent with akinetic rigid disorders in other brain injuries (vascular, toxic, medication induced, posttraumatic and post-encephalitic parkinsonism) [1], [2], [4]. This article discusses in details the clinical role of dopamine agonists, particularly pramipexole, in modern clinical practice.

\section{Modern conceptions of pathogenesis of Parkinson's disease}

Parkinsonism is known long since, the first information about treatment of such diseases appeared in 2500-100 BC in Ayurveda's lists, which was preserved to the present time, where there was a proposition to use the plant namely Magic Velvet Bean (mucuna pruriens), which consists of dopaminergic substrate. Some symptoms of parkinsonism were described by Galen in II century and Leonardo da Vinci in XV century. The first clinical description was made by English physician James Parkinson in "An Essay on the Shaking Palsy" published in 1817. Detailed clinical analysis of pathology complex was made in 1879 by French famous neurologist Jean-Martin Charcot. Except the trembling, difficulties with walking and general constraint admitted in due time by Parkinson as muscular feebleness. He introduced the concept of bradykinesia and proposed also a new name - Parkinson's disease. Among the movement disorders such syndromes are observed: paralysis, paresis, akinetic hypertonic and hyperkinetic hypotonic variants of movement disorders, ataxia, sudden falling and epileptic syndrome. Among them, the part of parkinsonism only akinetic hypertonic variants of movement disorders, which contain hypokinesia, muscle rigidity, tremor (especially in a resting state) and postural

\footnotetext{
* Corresponding author: Anatolii Tsarkov
} 
disorders. Muscle hypertonia and hypokinesia deprives the patient's possibility of coordinating movements and tremble makes big obstacles of self-care and professional activity. The symptoms of PD often are not limited only to movement disorders. Autonomic dysfunctions which are observed in patients with parkinsonism, provoke discomfort and embarrassment. Because of complexity of brain's pathobiochemistry, the dynamic balance is removed to the predominance of parasympathetic tone of autonomic nervous system.

Schizophrenia is a common psychiatric disorder in the practice of a psychiatrist. The main means in the treatment of this disease are neuroleptics. Although treatment for schizophrenia is effective, side effects such as movement disorders are common [5], [6], [7]. In this case, the disorder will be called symptomatic parkinsonism.

Typical symptoms of PD are arterial hypotonia and constipation as results of an autonomic dysfunction. Usually movement disorders are accompanied with hyperseborrhea ("oily face") especially on the forehead, sides of the nose, scalp and eyebrows, hypersalivation (sialorrhoea), arthrosis of fine joints, nails fragility, osteoporosis and weight loss. Disorders of autonomic regulation can be paroxysmal, mainly in the form of vago-insular and mixed crisis, but often there are cases of benign paroxysmal positional vertigo (BPPV), diffuse hyperhidrosis, cardialgia and frequent urination.

PD symptoms can affect mouth, teeth and jaw health, sometimes complicating dental treatment [8]. PD-related rigidity, tremor and dyskinesia can make it hard to brush one's teeth [8]. These symptoms can also cause cracked teeth, tooth wear, changes in the fit and wear of dentures and tooth grinding [8]. Symptoms such as fatigue, anxiety and tremor can make the commute to appointments, sitting still in the dentist's chair or opening the mouth wide is challenging [9]. Too much saliva can lead to a fungal infection at the corners of the mouth. By contrast, too little saliva or dry mouth increases the risk of cavities [8], [9].

Approximately $85 \%$ of patients have mental impairments. Often there are the symptoms of depression in such patients [2], [10], [11], [12]. Expressiveness of motive symptoms is dependent on psychical and emotional state of a patient: tremor increases emotional tension and diminishes at the resting state. Slowness of thought (bradyphrenia) in combination with the depression leads to severe impairment of cognitive functions and high risk of developing dementia. The symptoms associated with PD are presented in the Table 1 and differential diagnostic features of essential tremor and PD are presented in the Table 2.

Table 1 Symptoms associated with Parkinson's disease

\begin{tabular}{ll}
\hline Motor symptoms & Non-motor symptoms \\
\hline $\begin{array}{l}\text { Tremor, bradykinesia, rigidity, postural } \\
\text { instability }\end{array}$ & $\begin{array}{l}\text { Cognitive impairment, bradyphrenia, tip-of-the } \\
\text { tongue (word finding) phenomenon }\end{array}$ \\
Hypomimia, dysarthria, dysphagia, sialorrhoea & $\begin{array}{l}\text { Depression, apathy, anhedonia, fatigue, other } \\
\text { behavioural and psychiatric problems }\end{array}$ \\
$\begin{array}{l}\text { Decreased arm swing, shuffling gait, festination } \\
\text { difficulty arising from chair, turning in bed }\end{array}$ & $\begin{array}{l}\text { Sensory symptoms: anosmia, ageusia, pain } \\
\text { (shoulder, back), paresthesia }\end{array}$ \\
$\begin{array}{l}\text { Micrographia, cutting food, feeding, } \\
\text { hygiene, slow activities of daily living }\end{array}$ & $\begin{array}{l}\text { Dysautonomia (orthostatic hypotension, } \\
\text { constipation, urinary and sexual dysfunction, } \\
\text { abnormal sweating, seborrhea), weight loss }\end{array}$ \\
$\begin{array}{l}\text { Glabellar reflex, blepharospasm, dystonia, striatal } \\
\text { deformity, scoliosis, camptocormia (bent spine } \\
\text { syndrome) }\end{array}$ & $\begin{array}{l}\text { Sleep disorders (REM behaviour disorder, vivid } \\
\text { dreams, daytime drowsiness, sleep fragmentation, } \\
\text { restless legs syndrome) }\end{array}$ \\
\hline
\end{tabular}


Table 2 Features differentiating essential tremor from Parkinson's disease

\begin{tabular}{|c|c|c|}
\hline Feature & Essential tremor & Parkinson's disease \\
\hline Age at onset (years) & $10-80$ & $55-75$ \\
\hline Family history & ++ & $+/-$ \\
\hline Tremor frequency (Hz) & $5-10$ & $4-6$ \\
\hline Tremor characteristics & Flexion-extension & Supination-pronation \\
\hline Rest & Decreases & Increases \\
\hline Action & Increases & Decreases \\
\hline Mental concentration & Increases & Decreases \\
\hline Writing & Increases (tremulous) & Decreases (micrographia) \\
\hline Walking & Decreases & Increases \\
\hline Alcohol & Decreases & - \\
\hline Postural tremor & Without latency & Re-emergent \\
\hline Kinetic tremor & Yes & $+/-$ \\
\hline Limb tremor & Symmetric & Asymmetric \\
\hline Distribution other than limbs & Head, voice & Face, jaw, lips, chin \\
\hline $\begin{array}{l}\text { Neuroimaging (dopaminergic } \\
\text { system) }\end{array}$ & Mild dopaminergic deficit & Marked dopaminergic deficit \\
\hline Mid-brain sonography & Mild hyper-echogenicity & Marked hyper-echogenicity \\
\hline Neuropathology & $\begin{array}{l}\text { Mild cerebellar degeneration, } \\
\text { Lewy bodies in the substantia } \\
\text { nigra, brainstem and cerebellum } \\
\text { some cases }\end{array}$ & $\begin{array}{l}\text { Nigrostriatal degeneration, Lewy } \\
\text { bodies }\end{array}$ \\
\hline Treatment & $\begin{array}{l}\text { Alcohol, beta-blockers, } \\
\text { primidone, topiramate, } \\
\text { gabapentin, botulinum toxin, } \\
\text { deep brain stimulation }\end{array}$ & $\begin{array}{l}\text { Anticholinergics, amantadine, } \\
\text { dopaminergic drugs, deep brain } \\
\text { stimulation }\end{array}$ \\
\hline
\end{tabular}

\section{Principles of treatment of PD}

Degree of severity of the symptoms of PD is directly connected with the level of dopamine in nigrostriatal brain's system and it directly influences the choice of medications. In initial stage of the disease, the deficit of dopamine is expressed marginally, in spite of the degeneration of a significant part of substantia nigra. Preserved cells can provide the adequate level of dopamine through the increase of synthesis and the secretion of dopamine. This phase of PD can be considered partially compensated, that is why for the treatment we need the medicine that is able to "normalize" dopamine metabolism. Besides that, we need medicine, which can slack the degeneration of neurons and the progress of disease: so, we need the medicine, which has neuroprotective effects.

It is a general fact, that all the drugs used for PD treatment are labelled as the drugs for symptomatic treatment. Treatment of each patient should be individualized based on the severity of individual symptoms of the disease, the degree of functional maladjustment, age, level of activity of the necessary functionality, as well as the presence of side effects of therapy. For the patients, younger than 70 years which have the predominance of symptoms of rigidity and akinesia, it is recommended to start the treatment with inhibitor MAO-B - selegiline, amantadine or dopamine agonists (DAs). Anticholinergics are more effective for Idiopathic type of PD predominantly with tremor (tremor type of PD). In complex application of the mentioned medications levodopa is added only when there is no sufficient therapeutic effect. In patients, older than 70 years start of immediate treatment with small dose of levodopa is recommended, as in the elderly, other groups of medications are less effective and often cause somatic and mental side effects. 


\section{The relevance of neuroprotection in the PD treatment}

The possibilities of modern neurology in modification of progress of PD, prevention or deceleration of pathological process, prevention of some phases of disease or reverse progression of neurodegenerative changes - all that from clinical positions determine "neuroprotection" remains very important. The strategy of neuroprotection must be implemented in early or even presynaptic (latent) phase of neurodegenerative process, because of clinical manifestation forms of PD are connected with atrophy of $60-80 \%$ of dopaminergic nigral neurons and with catastrophic decline of the dopamine level in nigrostriatal dopaminergic neurons and cholinergic nerve endings [13]. It is actualized the developing of adequate biomarkers of PD - neuroimaging, neurophysiological, biochemical, molecular, genetic and others, which would be informative in early terms of the disease.

It is also believed that a therapeutic dose of Omega-3 fatty acids can help patients with PD [14]. Omega-3 fatty acids have a unique ability to cross the blood-brain barrier, which most drugs do not [15]. They can also increase dopamine levels and reduce inflammation in the brain [14]. For example, a subspecies of omega-3, eicosapentaenoic acid (EPA), has been shown to reduce the severity of motor disorders and neuroinflammation in animal models [14].

\section{The role of dopamine agonist in the PD treatment}

A significant achievement in PD therapy became after widespread use of dopamine agonists (DA). Although this class of antiparkinsonian medications are known since the early 1970s, practical importance is acquired only with the advent of the new generation of drugs such as pramipexole [16], [17], [18]. Results of numerous studies have shown that in the early stages of the disease DAs are capable of a fairly long period of delay prescribing of levodopa and limit its dose, in a late - to correct motor fluctuations and dyskinesia [19], [20], [21], [22].

Pramipexole is highly selective non-ergot dopamine agonist, mainly operating in the D3 subtype of dopamine D2 receptors, is a synthetic amino-benzothiazole derivative. The period of its half-life ranges from 8 hours (young people) and 12 hours (elderly). Reasonably to start pramipexole therapy with a sub-therapeutic dose ( $0.125 \mathrm{mg} /$ day), followed by its increase every 5-7 days to achieve personal well-tolerated dose and when we can see the therapeutic effect. In patients with PD pramipexole is effective both as monotherapy and in combination with levodopa.

Combination therapy in most cases can achieve a good result in the appointment of smaller doses, which reduces the potential risk of side effects of each drug. On the other hand, polypharmacology (a combination of three or more pharmaceutical agents) is undesirable because may contribute to the manifestation of side effects.

Levodopa and pramipexole complement each other and are often prescribed together to maximize efficiency and to minimize any side-effects. Perhaps this is due to a short half-life of levodopa, which when used as monotherapy, often provokes dyskinesia and other motor complications.

Pramipexole has a sufficiently long half-life and provides a long-lasting, close to the physiological conditions of DA stimulation, prevents or reduces the expression of developed motor fluctuations and drug-related dyskinesia related to the fluctuations in the level of levodopa concentration in the blood and changes in the sensitivity of dopamine receptors.

New pathophysiological concept suggests that non-physiological stimulation of the dopamine receptors trigger a cascade of new pathological processes: dysregulation of dopaminergic and non-dopaminergic receptors of striatum, the formation of abnormal intracellular pulses in striatal neurons and basal ganglia, which ultimately leads to motor fluctuations and dyskinesia. Pulsatile dopamine receptor stimulation leads to the induction of gene expression of various changes in striatal neurons. This data is the basis for DAs as a first-line therapy in younger patients and elderly patients without decompensated comorbidities.

One of the important argument for the advisability of the beginning of PD therapy with DA is their possible neuroprotective effect. The interaction between genetic and exterior factors is a base of neurodegenerative process in the case of PD. Pathophysiological mechanisms of neurodegeneration implemented via oxidative stress, formation of free radicals, the development of mitochondrial and proteasomal dysfunction and inflammation.

\section{Efficacy of pramipexole}

According to the recommendations of The European Federation of Neurological Societies and European section of the Movement Disorder Society (2013), the drug of choice must depend on the phase of the disease, the age of a patient, 
predominant clinical manifestations, level of cognitive functions of the patient, severity of motor disorders with risk of development of motor and neuropsychiatric complications in the background of the prescribed therapy [23]. The authors of the guide recommend pramipexole in oral or transdermal forms for patients in early stage of PD and for patients who previously have not taken other prescribed treatment [23]. Clinical efficacy of pramipexole was proved through numerous short and long-term randomized double-blind placebo-controlled trials in the treatment of early and advanced stages of PD. During monotherapy with pramipexole in early stage of PD degree of movement disorders expression was reduced. The score of Unified Parkinson's disease rating scale (UPDRS, part III) was lowering on the average to $25 \%$ in comparison with the deterioration to $6.9 \%$ in the group of patients, who were taking placebo [23].

Pramipexole showed to be effective in reducing the duration of the "switched off" period [21], [24], [25]. It is important to note that when high doses of DA are applied the need for levodopa therapy usually reduced and as a result, dyskinesia decreased [21].

In a direct comparison of the effectiveness of pramipexole and levodopa as initial PD therapy pramipexole significantly reduced the development of motor fluctuations and medication-induced dyskinesia. Initial pramipexole therapy for 2 and 4 years reduced patient's risk of motor fluctuations in early PD (phenomena of dose response exhaustion and "switching on-off") at 30\% compared to levodopa and medication-induced dyskinesia at 50\%. However, the overall improvement in the UPDRS scale after 2 years of therapy was more pronounced in the levodopa group, and score on the scale of quality of life did not differ between the two groups [23], [24].

In a double-blind placebo controlled test, where 291 patients were included, the effectiveness of pramipexole by motor fluctuations was evaluated in comparison with placebo [22]. Except the lowering of motor fluctuations, pramipexole demonstrated the effectiveness concerning the duration and the severity of "off-periods" in comparison with placebo, and also allowed to lower the levodopa dose. Documented unwanted side effects did not differ from those of other drugs in this group. Except the effectiveness in rigidity and akinesia treatment, pramipexole was lowering the expression of tremor, which was demonstrated during the research with the participation of 16 patients with the early phases of PD (11 participants were taking pramipexole, 5 were taking placebo) [23]. The positive dynamics was marked already during the phase of taking $0,75 \mathrm{mg} /$ day $(35 \%$ had the tremor lowering, $22 \%$ had the akinesia lowering according to the UPDRS during "on-periods"). The patients, who were taking $4,5 \mathrm{mg} /$ day, demonstrated $61 \%$ of tremor lowering and $65 \%$ of rigidity and akinesia lowering. In the ones, who were taking placebo, the improvement of these symptoms was not observed. The results of research demonstrated, that pramipexole can be useful for the patients in the late phases of PD, helping the lowering of motor fluctuations expressions and dyskinesia expressions [23]. The medicine was giving the most important influence to the phenomenon of exhaustion of the end of dose of levodopa and the dyskinesia of a "off period". At the same time, the positive influence on the sudden "switching" and other variants of dyskinesia was less stable. Depression occurs in approximately $40 \%$ of patients with the PD and influences negatively on their life quality [23]. Significant advantage of pramipexole, which is also mentioned in the EFNS / MDS-ES manual (2013), is its antidepressant effect [23]. In a small study during 8 months was mentioned the reduce of anxiety and depressive symptoms in the course of pergolide and pramipexole [26]. Antidepressant effect of pramipexole in the cases of PD also was confirmed in the placebo-controlled study, where the improvement was explained by the immediate antidepressant effect [24].

One disadvantage of pramipexole treatment should be mentioned. Pramipexole and other direct dopamine agonist medications have been implicated in the development of impulsive behavior such as pathological gambling [27]. Gambling addiction is a pathological addiction to gambling; is frequent repeated episodes of participation in gambling, which dominate in a person's life and lead to a decrease in social, professional, material and family values. [28]. This side effect has to be carefully monitored while patient and her/his relatives need to be warned about this negative side effect.

Finally, pramipexole works as monotherapy in unipolar depression, but in bipolar disorder it should be taken in concert with a mood stabilizer [29]. That could be lithium, valproate, or, in bipolar II patients, lamotrigine [29], [30], [31], [32]. It can also be taken with an antipsychotic, as most of these antagonize a different dopamine receptor (D2). A few antipsychotics might synergize D3 effects of pramipexole, such as aripiprazole, brexpiprazole, cariprazine, and lumateperone, which are partial D3 agonists [29].

\section{Conclusion}

The treatment of PD with pramipexole ensures very good control of movement disorders and the efficacy on any stage of the disease. Pramipexole is able to control tremor, hypokinesia, rigidity and postural instability. It also corrects a wide range of neuropsychiatric symptoms including depression and anxiety. The correction of different symptoms by 
using monotherapy of pramipexole has the other great advantage in PD therapy. Nevertheless, in the case of necessity of the prescription of pramipexole in combination with other antiparkinsonian medication, the daily dose of levodopa can be reduced without the loss of pharmacotherapy effects. During the treatment with pramipexole of the early and late stages of PD, we may observe the reduction of expression of cognitive and affective (mood) disorders, the improvement of the quality of life and social functioning. Pramipexole may be prescribed in a case of unipolar depression as monotherapy or with combination of mood stabilizer for bipolar disorder.

\section{Compliance with ethical standards}

\section{Acknowledgments}

We acknowledge the valuable inputs given by Professor Trevor Kaile (Dean, School of Medicine, University of Zambia UNZA), Doctor Elliot Kafumukache (Acting Dean, School of Medicine, University of Zambia - UNZA) and Doctor Ravi Paul (Head of Department, Department of Psychiatry, University of Zambia - UNZA) in the preparation of this paper.

\section{Disclosure of conflict of interest}

There is no conflict of interest.

\section{References}

[1] Koller W. (2014). How accurately can Parkinson's disease be diagnosed? Neurology, 42(1), 4-5.

[2] Jankovic J. (2008). Parkinson's disease: clinical features and diagnosis. J Neurol Neurosurg Psychiatry, 79, 368376.

[3] Tsarkov A and Petlovanyi P. (2019). Neuropsychiatric Aspects of a Common Problem: Stroke. European Journal of Medical and Health Sciences, 1(3).

[4] Barone P, Poewe W and Tolosa E. (2010). Efficacy of double-blind, placebo-controlled pramipexole against depression in Parkinson's disease. Abstracts of the Movement Disorders Society's Thirteenth International Congress of Parkinson's disease and movement disorders, suppl, 1, 291.

[5] Petlovanyi P and Tsarkov A. Child Schizophrenia: Theory and Practice.

[6] Anatolii T, Patrick M and Petro P. (2020). Uncommon presentation: Folie à deux (Case study). World Journal of Advanced Research and Reviews, 6(3), 043-9.

[7] Tsarkov A, Msoni P and Petlovanyi P. (2020). Uncommon presentation: Folie à deux (Case study). World Journal of Advanced Research and Reviews, 6(3), 43-49.

[8] Bakke M, Larsen SL, Lautrup C and Karlsborg M. (2011). Orofacial function and oral health in patients with Parkinson's disease. European journal of oral sciences, 119(1), 27-32.

[9] Phiri C, Tsarkov A, Petlovanyi P and Lingenda G. Factors Contributing To Oral Diseases and Treatment Needs amongst Mental Patients at Chainama Hills College Hospital, Lusaka, Zambia.

[10] Mierau J and Schingnitz G. (1992). Biochemical and pharmacological studies on pramipexole, a potent and selective dopamine Da receptor agonist. Eur. J. Pharmacol, 161-170.

[11] Petlovanyi P and Tsarkov A. (2017). Depressive Disorder in Child Psychiatric Practice: A Case Report. Health Press Zambia Bull, 1(5), 9.

[12] Tsarkov A and Petlovanyi P. Depressive Disorder in Child Psychiatric Practice: A. The Health Press, 9.

[13] Shapira AH. (2007). Treatment options in the modern management of Parkinson disease. Arch Neurol, 64, 10831088.

[14] Bousquet M, Calon F and Cicchetti F. (2011). Impact of omega-3 fatty acids in Parkinson's disease. Ageing research reviews, 10(4), 453-63.

[15] Tsarkov A and Petlovanyi P. (2017). Omega-3 Fatty Acids as an Alternative Treatment for Children with Attention Deficit Hyperactivity Disorder. Imperial Journal of Interdisciplinary Research (IJIR), 3, 1378-80.

[16] Goets CG, Blasuci L and Stebbins GS. (1999). Switching dopamine agonists in advanced Parkinson's disease. Is rapid titration preferable to slow? Neurology, 52, 1227-1229. 
[17] Olanow CW, Schapria A and Rascol O. (2000). Continuous dopamine-receptor stimulation in early Parkinson's disease. Trends. Neurosci, 23, 117-126.

[18] Hobson DE, Lang AE and Martin WR. (2002). Excessive daytime sleepiness and sudden onset sleep in Parkinson's disease, 287(4), 455-463.

[19] Rinne U, Bracco F and Chouza C. (1998). Early treatment of Parkinson's disease with cabergoline delays the onset of motor complications. Results of double-blind study. Drugs, 55(1), 72-81.

[20] The Parkinson Study Group. (2000). Pramipexole vs levodopa as initial treatment for Parkinson disease. JAMA, 284, 231-238.

[21] Weiner WJ and Reich SG. (2008). Agonist or levodopa for Parkinson disease: ultimately, it doesn't matter; neither is good enough. Neurology, 71(7), 470-471.

[22] Lieberman A, Ranhosky and Korts D. (1997). Clinical evaluation of pramipexole in advanced Parkinson's disease. Neurology, 49, 162-163.

[23] Ferreira JJ, Katzenschlager R and Bloem BR. (2013). Summary of the recommendations of the EFNS/MDS-ES review on therapeutic management of Parkinson's disease. Eur. J. Neurol, 20, 5-15.

[24] Rektorová I, Rektor I and Bares M. (2003). Pramipexole and pergolide in the treatment of depression in Parkinson's disease: a national multicenter prospective randomized study. Eur. J. Neurol, 10(4), 399-406.

[25] Katzenschlager R, Sampaio C and Costa J (2003). Cochrane Database Syst. Rev.

[26] Manson AJ, Hanagasi H and Turner K. (2002). Intravenous apomorphine therapy in Parkinson's disease: clinical and pharmacokinetic observations. Brain, 124, 331-340.

[27] Spengos K, Grips E and Karachalios G. (2006). Reversible pathological gambling under treatment with pramipexole. Nervenarzt, 77, 958-960.

[28] Tsarkov A and Petlovanyi P. (2017). Pathological Gambling: The Old Problem of the Modern World. Imperial Journal of Interdisciplinary Research, 3(8), 216-21.

[29] Aiken C. (2020). An Underutilized Option for Bipolar Depression. Psychiatric Times.

[30] Tsarkov A and Petlovanyi P. The Role of Lamotrigine in the Treatment of Bipolar Depression.

[31] Tsarkov A and Petlovanyi P. Bipolar disorder in child psychiatric practice.

[32] Tsarkov A and Petlovanyi P. (2016). Bipolar Disorder in Child Psychiatric Practice: A Case Report. Medical Journal of Zambia, 43(1), 41-6.

\section{How to cite this article}

Tsarkov A and Petlovanyi P. (2020). Use of pramipexole in neuropsychiatry. World Journal of Advanced Research and Reviews, 7(2), 82-88. 